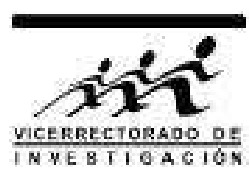

\title{
Estudio de la quinua, Chenopodium quinoa Willd, como fertilizante natural alternativo, mediante métodos nucleares analíticos
}

\author{
R. L. Soto*1, O. M. Chumpitaz ${ }^{2}$, E. Torres ${ }^{1}$, J. Rojas ${ }^{1}$, J. C. Llópiz ${ }^{3}$ y S. Szegedi \\ 1 Facultad de Ciencias Físicas, Universidad Nacional Mayor de San Marcos, Lima, Perú \\ 2 Facultad de Química e Ing. Química, Universidad Nacional Mayor de San Marcos, Lima, Perú \\ ${ }^{3}$ Instituto de Materiales y Reactivos, Universidad de la Habana, La Habana, Cuba \\ ${ }^{4}$ Institute of Experimental Physics, Kossuth University, Debrecen, Hungary
}

Recibido 25 marzo 2015 - Aceptado 10 julio 2015

\begin{abstract}
Con base en la aplicación de los métodos analíticos de caracterización multielemental de las muestras orgánicas, tales como la fluorescencia de rayos $\mathrm{X}$, el análisis por activación neutrónica y el análisis químico de Coleman, se sugiere la posibilidad de uso de varias partes no comestibles de la planta denominada quinua, Chenopodium quinoa Willd, como fertilizante natural alternativo para este cultivo.

Palabras claves: Métodos nucleares analíticos, Chenopodium quinoa willd, agricultura ecológica, abonos verdes, fertilizantes nitrogenados.
\end{abstract}

\section{Study of quinoa, Chenopodium quinoa Willd, as an alternative na- tural fertilizer by nuclear analytical methods}

On the base of the application of multielemental analytic methods of characterization of organic samples, such as the fluorescence of X-rays, neutron activation analysis and Coleman chemical analysis, the possibility of use of several parts of the plant named Quinua, Chenopodium quinoa Willd is suggested as alternative natural fertilizer for this crop.

Keywords: Nuclera analytical methods, Chenopodium quinoa willd, ecological farming, green manures, nitrogenated fertilizer.

La planta de quinua, Chenopodium quinoa Wild, es un cultivo originario de la región andina de América del Sur donde existe un ecosistema especialmente apropiado para el cultivo de esta especie de la cual se han documentado varios estudios que establecen su composición en aminoácidos, incluídos los diez esenciales para el ser humano entre los que destaca la lisina de vital importancia para el desarrollo de las células cerebrales, los procesos de aprendizaje, memorización y raciocinio, así como para el crecimiento físico [1,2]. Los granos de este pseudocereal, proporcionan minerales y vitaminas tales como la A, C, D, B1, B2, B6, ácido fólico, niacina, calcio, hierro y fósforo sin que su ingestión suponga la inclusión de colesterol ni de gluten en la dieta, además, sustituye muy bien las proteínas de origen animal estando libre de grasas, ácido úrico y toxinas propias de los alimentos con esta procedencia [3,4]. Considerando las virtudes de la quinua y las necesidades alimenticias de la creciente población global, varias instituciones, entre ellas la Organización para la Alimentación y la Agri-

*Isoto1953@hotmail.com 
cultura, FAO [5], la Organización Mundial de la Salud, OMS [6], el Instituto Interamericano de Cooperación para la Agricultura, así como varios institutos destinados a las investigaciones agrícolas, lo consideran un alimento único por su alto valor nutricional [7,8] y han propendido investigaciones encaminadas a su mejoramiento ya que crece de forma rústica entre los 2500 a $4000 \mathrm{~m}$ de altura y resiste adversidades climatológicas de bajas temperaturas, desde $-4.0{ }^{\circ} \mathrm{C}$ a $-7.8{ }^{\circ} \mathrm{C}$ en la etapa de la floración y de $-10.4{ }^{\circ} \mathrm{C}$ en estado de grano lechoso [7]. En cuanto a los suelos, admite tierras áridas y semiáridas, lo que determina sus ventajas para ser cultivada en el Altiplano andino como tradicionalmente ha sido realizado por los pueblos indígenas [9], para los que constituye una fuente primordial de alimentación y este detalle ha impulsado la necesidad de que se realicen investigaciones a fin de potenciar un incremento en las producciones pero sin conducir a un desmedido uso de los fertilizantes químicos nitrogenados. En el Congreso Internacional sobre la Quinua celebrado en 2013, en el Simposio Latinoamericano de Nutrición, se ha puesto de manifiesto la necesidad de establecer la caracterización composicional de nitrógeno y potasio en la quinua. En el mercado actual, el precio de la quinua producida por fertilizantes naturales orgánicos es mucho mayor al precio de la quinua producida con fertilizantes químicos.

El presente trabajo muestra resultados cuantitativos sobre el contenido de nitrógeno y potasio obtenidos a través de los métodos nucleares analíticos aplicados a diferentes partes de la planta con el propósito de aportar criterios que favorecen su uso como fertilizante natural en el cultivo de la propia.

\section{Clasificación de las muestras.}

Se ha realizado el cultivo de la quinua con tres criterios que denominamos, T-1, como el primer esquema de fertilización en la que los suelos fueron tratados con $80 \mathrm{~kg} / \mathrm{ha}$ de $\mathrm{N}$ al ser sembrada la planta; T-2, el segundo esquema de fertilización, que representa el empleo de $40 \mathrm{~kg} /$ ha de $\mathrm{N}$ aplicados al momento de la siembra y nuevamente se aplica la misma cantidad, a los 60 días; $\mathbf{T}-3$, es el control en el que no se utiliza fertilizante alguno [10]. La selección de las muestras toma en cuenta el estadio de desarrollo de las plantas, para lo cuales es suficiente realizar los análisis a los 60, 75, 100 y 174 días, como aparece en la Tabla 1 .

\begin{tabular}{|c|c|c|c|c|c|}
\hline $\begin{array}{l}\text { Parte de la } \\
\text { planta }\end{array}$ & Esquema & $\begin{array}{c}\text { 1ra Epoca } \\
60 \text { días }\end{array}$ & $\begin{array}{c}\text { 2da Epoca } \\
75 \text { días }\end{array}$ & $\begin{array}{l}\text { 3ra Epoca } \\
100 \text { días }\end{array}$ & $\begin{array}{c}\text { 4ta Epoca } \\
174 \text { días }\end{array}$ \\
\hline Hoja & T-1 & M-01 & M-02 & M-03 & \\
\hline Tallo & T-1 & M-04 & M-05 & M-06 & \\
\hline Raíz & $\mathrm{T}-1$ & M-07 & M-08 & M-09 & \\
\hline Flor & $\mathrm{T}-1$ & & & M-19 & \\
\hline Grano & $\mathrm{T}-1$ & & & & M-16 \\
\hline Hoja & $\mathrm{T}-2$ & $\mathrm{M}-10$ & M-11 & M-12 & \\
\hline Tallo & $\mathrm{T}-2$ & M-13 & M-14 & M-15 & \\
\hline Raíz & $\mathrm{T}-2$ & M-20 & M-21 & M-22 & \\
\hline Flor & $\mathrm{T}-2$ & & & M-18 & \\
\hline Grano & $\mathrm{T}-2$ & & & & M-17 \\
\hline Hoja & $\mathrm{T}-3$ & M-23 & M-24 & M-25 & \\
\hline Tallo & $\mathrm{T}-3$ & M-26 & $M-27$ & $M-28$ & \\
\hline Raíz & T-3 & M-29 & M-30 & M-31 & \\
\hline Flor & T-3 & & & M-32 & \\
\hline Grano & T-3 & & & & M-33 \\
\hline
\end{tabular}

Tabla 1: Clasificación de las muestras estudiadas.

Las muestras fueron preparadas con el doble propósito de ser analizadas mediante el método de los estándares internos [11, 12], para lo cual se adicionan cantidades conocidas del compuesto químico $\mathrm{KNO}_{3}$ cuyo número atómico promedio no es muy alto con respecto a esta magnitud para el caso de las muestras de procedencia orgánica como las que se emplean en el presente trabajo, con ello, se establece una ecuación de 
calibración que permite la determinación de la cantidad de potasio [13], mediante la aplicación del método de fluorescencia de rayos $X$ considerando previamente el comportamiento del número atómico promedio de las muestras estudiadas para cuantificar el efecto de la matriz orgánica.

El método citado fue utilizado también para establecer cuantitativamente las interferencias que el elemento potasio produce durante la aplicación del método de activación con neutrones de $14 \mathrm{MeV}$ [13], al determinar la cantidad de nitrógeno presente; de esta forma se obtienen los valores de la concentración de potasio y nitrógeno para cada muestra mediante la combinación de ambos. Con base en lo anterior las muestras fueron confeccionadas con un diámetro de $20 \mathrm{~mm}$, masa de 700 $\mathrm{mg}$, espesor entre 2.5 y $3 \mathrm{~mm}$ y un proceso de secado a una temperatura de $80{ }^{\circ} \mathrm{C}$ durante dos horas. Dado el carácter no destructivo de los métodos aplicados, se realizó el número requerido de mediciones para garantizar la fiabilidad estadística de los resultados dentro del $95 \%$ de intervalo de confianza.

\section{Resultados y discusión}

Se empleó el análisis por fluorescencia de rayos $X$ con el propósito de determinar otros elementos presentes en las muestras de lo que se concluyó la presencia, dentro de los límites de sensibilidad de este método, $\mathrm{C}(\mathrm{K}) \pm 8 \%$, de potasio, hierro, zinc, bromo, estroncio, molibdeno y vanadio. La Tabla 2 muestra el resultado de la determinación de la concentración de potasio en las muestras estudiadas.

\begin{tabular}{cccc||cccc}
\hline Muestra & Parte & $C_{K}(\%)$ & $C_{K}(\mathrm{mg} / 100 \mathrm{~g})$ & Muestra & Parte & $C_{K}(\%)$ & $C_{K}(\mathrm{mg} / 100 \mathrm{~g})$ \\
\hline M-01 T-1 & Hoja & 5.43 & 0.776 & M-18 T-2 & Flor & 2.71 & 0.387 \\
M-02 T-1 & Hoja & 5.53 & 0.790 & M-19 T-1 & Flor & 2.37 & 0.339 \\
M-03 T-1 & Hoja & 4.34 & 0.620 & M-20 T-2 & Raíz & 2.62 & 0.374 \\
M-04 T-1 & Tallo & 5.44 & 0.777 & M-21 T-2 & Raíz & 2.61 & 0.373 \\
M-05 T-1 & Tallo & 6.34 & 0.906 & M-22 T-2 & Raíz & 1.13 & 0.161 \\
M-06 T-1 & Tallo & 1.74 & 0.249 & M-23 T-3 & Hoja & 4.76 & 0.680 \\
M-07 T-1 & Raíz & 2.16 & 0.309 & M-24 T-3 & Hoja & 2.83 & 0.404 \\
M-08 T-1 & Raíz & 1.58 & 0.226 & M-25 T-3 & Hoja & 3.45 & 0.493 \\
M-09 T-1 & Raíz & 1.28 & 0.183 & M-26 T-3 & Tallo & 4.88 & 0.697 \\
M-10 T-2 & Hoja & 5.17 & 0.739 & M-27 T-3 & Tallo & 2.62 & 0.374 \\
M-11 T-2 & Hoja & 6.12 & 0.874 & M-28 T-3 & Tallo & 1.20 & 0.171 \\
M-12 T-2 & Hoja & 4.78 & 0.683 & M-29 T-3 & Raíz & 2.97 & 0.424 \\
M-13 T-2 & Tallo & 5.36 & 0.766 & M-30 T-3 & Raíz & 2.25 & 0.321 \\
M-14 T-2 & Tallo & 3.82 & 0.546 & M-31 T-3 & Raíz & 0.93 & 0.133 \\
M-15 T-2 & Tallo & 1.84 & 0.263 & M-32 T-3 & Flor & 2.53 & 0.361 \\
M-16 T-1 & Grano & 0.56 & 0.080 & M-33 T-3 & Grano & 0.48 & 0.069 \\
M-17 T-2 & Grano & 0.46 & 0.066 & & & & \\
\hline
\end{tabular}

Tabla 2: Concentración de potasio para cada muestra.

Los resultados mostrados en la Tabla 2 concuerdan con lo reportado para el grano en estudios precedentes, donde se establece que la quinua aporta $0.697 \mathrm{mg} / 100 \mathrm{~g}$, mientras que [14 15] reportan la presencia de potasio entre 0.840 y $1.145 \mathrm{mg} / 100 \mathrm{~g}$ de materia seca. Cuantificada la presencia de potasio que se comporta como una interferencia en la determinación del nitrógeno median- te el análisis por activación neutrónica y de considerar una tabla de tiempos adecuada que suprima la presencia del fósforo activado que también interfiere en las mediciones señaladas, se estableció el contenido de nitrógeno en las muestras estudiadas, como aparece en la Tabla 3 


\begin{tabular}{ccccc||ccccc}
\hline Muestra & Parte & $\begin{array}{c}\mathrm{M}_{\mathrm{N}} \\
(\mathrm{mg} / 100 \mathrm{~g})\end{array}$ & $\begin{array}{c}\text { Error } \\
(\mathrm{mg} / 100 \mathrm{~g})\end{array}$ & $\begin{array}{c}\mathrm{M}_{\mathrm{N}} \\
(\%)\end{array}$ & Muestra & Parte & $\begin{array}{c}\mathrm{M}_{\mathrm{N}} \\
(\mathrm{mg} / 100 \mathrm{~g})\end{array}$ & $\begin{array}{c}\text { Error } \\
(\mathrm{mg} / 100 \mathrm{~g})\end{array}$ & $\begin{array}{c}\mathrm{M}_{\mathrm{N}} \\
(\%)\end{array}$ \\
\hline M-01 T-1 & Hoja & 36.24 & 5.33 & 5.61 & M-18 T-2 & Flor & 25.27 & 3.70 & 4.23 \\
M-02 T-1 & Hoja & 38.20 & 5.37 & 5.83 & M-19 T-1 & Flor & 21.40 & 3.06 & 2.96 \\
M-03 T-1 & Hoja & 31.53 & 4.59 & 4.79 & M-20 T-2 & Raíz & 19.59 & 2.76 & 2.94 \\
M-04 T-1 & Tallo & 37.90 & 5.44 & 5.72 & M-21 T-2 & Raíz & 16.29 & 2.35 & 2.37 \\
M-05 T-1 & Tallo & 39.92 & 5.80 & 5.95 & M-22 T-2 & Rá́z & 09.85 & 1.42 & 1.52 \\
M-06 T-1 & Tallo & 12.53 & 1.77 & 1.83 & M-23 T-3 & Hoja & 36.57 & 5.25 & 5.30 \\
M-07 T-1 & Raíz & 20.09 & 3.85 & 3.11 & M-24 T-3 & Hoja & 23.60 & 3.41 & 3.89 \\
M-08 T-1 & Raíz & 15.22 & 2.17 & 2.40 & M-25 T-3 & Hoja & 21.92 & 3.13 & 3.52 \\
M-09 T-1 & Raíz & 10.82 & 1.55 & 1.57 & M-26 T-3 & Tallo & 35.52 & 5.12 & 5.37 \\
M-10 T-2 & Hoja & 41.03 & 5.83 & 6.01 & M-27 T-3 & Tallo & 24.46 & 3.50 & 3.92 \\
M-11 T-2 & Hoja & 38.90 & 5.57 & 5.73 & M-28 T-3 & Tallo & 09.74 & 1.42 & 1.56 \\
M-12 T-2 & Hoja & 31.86 & 4.62 & 4.88 & M-29 T-3 & Raíz & 18.97 & 2.73 & 2.67 \\
M-13 T-2 & Tallo & 32.71 & 4.72 & 5.51 & M-30 T-3 & Rá́z & 12.74 & 1.86 & 2.12 \\
M-14 T-2 & Tallo & 33.33 & 4.74 & 5.33 & M-31 T-3 & Raíz & 09.90 & 1.47 & 1.53 \\
M-15 T-2 & Tallo & 11.19 & 1.62 & 2.58 & M-32 T-3 & Flor & 23.34 & 3.39 & 3.67 \\
M-16 T-1 & Grano & 13.11 & 2.00 & 2.11 & M-33 T-3 & Grano & 12.71 & 1.86 & 2.04 \\
M-17 T-2 & Grano & 16.53 & 2.62 & 2.73 & & & & & \\
\hline
\end{tabular}

Tabla 3: Determinación del contenido de N. Método relativo.

A partir de las determinaciones cuantitativas anteriores, se aprecia que independientemente del esquema de fertilización adoptado, T-1 mostrado en rojo y T-2 mostrado en verde, en la Fig. 1, la masa de nitrógeno (mg) decrece con la época o el estadio de crecimiento de la planta de quinua. Igual comportamiento se observa para el control, T-3 mostrado en naranja, como se muestra en la Fig. 1. De la propia figura se observa que aún considerando la reducción de este elemento en las raíces, los tallos, las hojas y las flores que son las partes que generalmente se desechan al término de la cosecha, la cantidad de nitrógeno es considerable. En consecuencia, es factible utilizar estos desechos mediante su transformación en abono orgánico [16, 17], generado por la propia producción de quinua que conduciría a incrementar el tiempo de fertilidad del terreno [18], para evitar el desgaste de la superficie cultivable [19] y para disminuir la erosión que perjudica el cultivo [20] y la calidad de la tierra. Similares comentarios y conclusiones pueden extenderse de la determinación de las cantidades de potasio en las muestras, mostradas en la tabla 2.

Aunque mayoritariamente la importancia del cultivo de la quinua radica en las propiedades nutritivas del grano [21], es significativo el valor del contenido de nitrógeno en las hojas tiernas, como se observa de la Fig. 2.

El resultado apuntado valida su utilización tanto pa- ra la alimentación de aves como de otros animales sin excluir al hombre, costumbre prácticamente olvidada en la que las hojas tiernas de la quinua se usaban como hortalizas Ilipccha o cchiwa. Como puede constatarse de la Tabla 2, la incorporación de esta parte de la planta a la dieta del poblador andino constituye una fuente de potasio que aportaría entre 0.620 y $0.790 \mathrm{~g}$ de potasio/ 100 $\mathrm{g}$ de hojas de quinua consumidas.

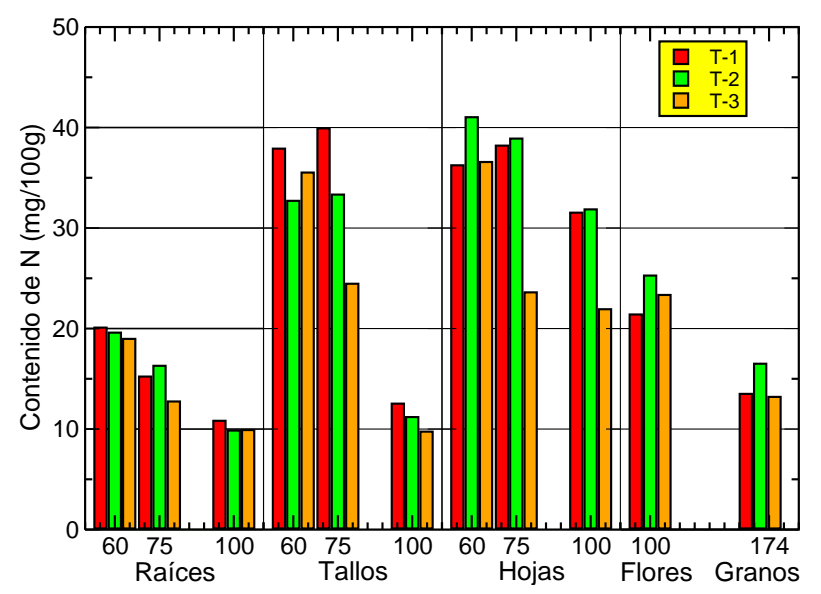

Figura 1: Comportamiento de la masa de nitrógeno para cada parte de la planta de quinua según su periodo vegetativo. Observe que los esquemas de fertilización están dados T-1 (rojo), T-2(verde) y T-3 (naranja) se refiere al control. 


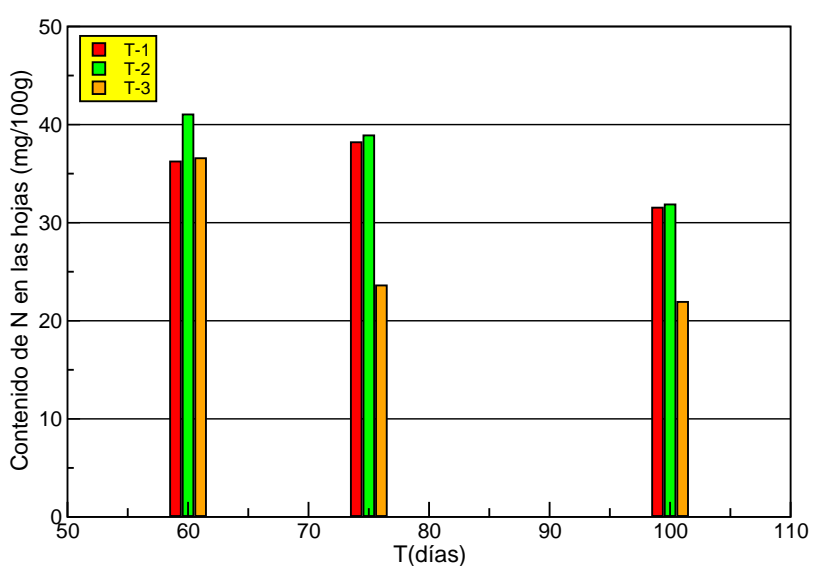

Figura 2: Cantidad de nitrógeno en las hojas de la quinua para los tres esquemas de fertilización, T-1, T-2 y T-3.
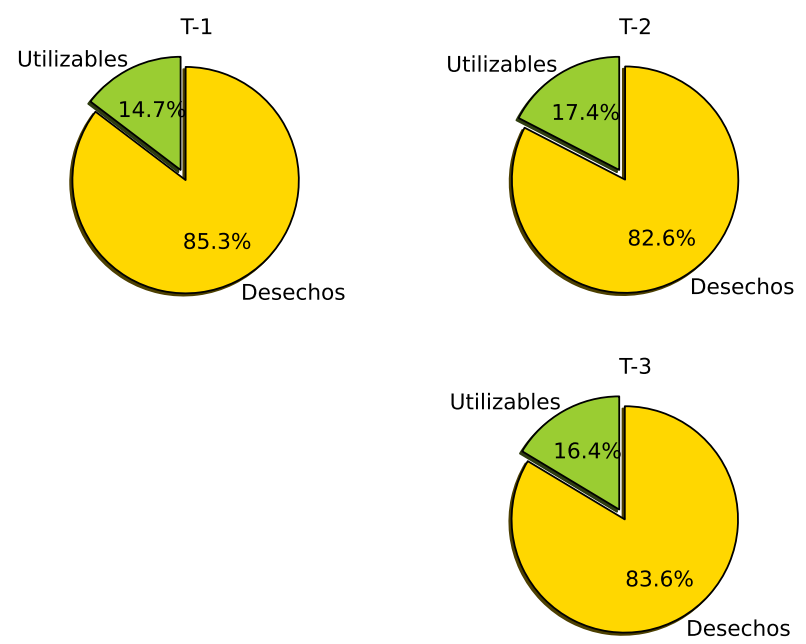

Figura 3: Desechos y utilizables expresados en términos de la masa de $\mathrm{N}(\%)$

Como en todo proceso productivo de tipo extractivo comercial en el que no se disponga de un plan de manejo adecuado de los recursos naturales, se produce un deterioro acelerado del medio ambiente con el con- siguiente impacto ambiental en los ecosistemas, frágiles por excelencia en todo el Altiplano andino, se hace necesario recurrir a una agricultura [22,23], que propenda la reincorporación de nutrientes y la disponibilidad de elementos a la par de materia orgánica que permita un manejo de los suelos con proyección sustentable. Como se observa en la Fig. 3, el cultivo de la quinua posibilita la utilización de los desechables de la cosecha. De la propia Fig. 3 puede apreciarse que aún en el caso de que el cultivo de quinua se realice sin la aplicación de fertilizante químico alguno, la contribución a la masa de nitrógeno por los desechables garantiza más del $80 \%$ en peso de este importante elemento si se utilizan en forma de abonos verdes las raíces, los tallos, las hojas y las flores o panojas una vez que la extracción del grano haya sido completada. Lo anterior contribuiría notablemente a la sostenibilidad de la actividad agrícola humana en correspondencia con el necesario equilibrio ambiental que supone la agricultura ecológica, orgánica o biológica. Similares resultados se obtienen al analizar la presencia de potasio en los desechables de la cosecha de quinua y si se toma en cuenta la identificación cualitativa hecha, puede asegurarse la revitalización de los suelos al incorporarse otros elementos como hierro, zinc, bromo, estroncio, molibdeno y vanadio [24.

Aunque el presente trabajo tiene por objetivo argumentar con resultados cuantitativos la factibilidad del uso de los desechables de la cosecha del cultivo de quinua en forma de abonos verdes o mixtos con adición de estiércol de diferentes procedencias, éste no puede desconocer el efecto que se produce sobre el contenido proteico del grano [25], medido a través de los valores de la masa de nitrógeno que alcanza, cuando se utilizan los esquemas de fertilización descritos en la sección sobre la clasificación de las muestras. La Tabla 4 muestra el porcentaje en peso de nitrógeno y las incertidumbres inherentes a los métodos analíticos utilizados; mientras que en la Tabla [5] aparece reportado el contenido proteico de los granos.

\begin{tabular}{cc|cc|cc|cc}
\hline Muestra & Esquema & $\begin{array}{c}\text { Método } \\
\text { absoluto } \\
\% \mathrm{~N}\end{array}$ & $\begin{array}{c}\text { Error } \\
\%\end{array}$ & $\begin{array}{c}\text { Método } \\
\text { relativo } \\
\% \mathrm{~N}\end{array}$ & $\begin{array}{c}\text { Error } \\
\%\end{array}$ & $\begin{array}{c}\text { Método químico } \\
\text { de Coleman } \\
\% \mathrm{~N}\end{array}$ & $\begin{array}{c}\text { Error } \\
\%\end{array}$ \\
\hline $\mathrm{M}-16$ & $\mathrm{~T}-1$ & 1.85 & 0.32 & 2.11 & 0.32 & 2.22 & 0.20 \\
$\mathrm{M}-17$ & $\mathrm{~T}-2$ & 2.24 & 0.39 & 2.73 & 0.43 & 2.70 & 0.20 \\
$\mathrm{M}-33$ & $\mathrm{~T}-3$ & 1.95 & 0.33 & 2.04 & 0.30 & 1.99 & 0.20 \\
\hline
\end{tabular}

Tabla 4: Comparación de los resultados obtenidos mediante el empleo de la activación neutrónica y el procedimiento químico de Coleman, para los tres esquemas de fertilización, siendo T-3 el control. 
De la propia Tabla 4 se aprecia que los resultados de la aplicación del análisis por activación neutrónica, (método relativo), presenta discrepancias porcentuales inferiores al $7 \%$ respecto del reconocido método químico de Coleman y de considerar la Tabla 5 , puede concluirse que el uso de fertilizantes nitrogenados como los empleados en este trabajo no mejora ostensiblemente el valor nutricional de los granos según el esquema de fertilización T-1.

\begin{tabular}{cccc}
\hline Muestra & $\begin{array}{c}\text { Esquema de } \\
\text { fertilización }\end{array}$ & $\begin{array}{c}\text { Contenido } \\
\text { proteico } \\
(\%)\end{array}$ & $\begin{array}{c}\text { Error } \\
(\%)\end{array}$ \\
\hline 16 & $\mathrm{~T}-1$ & 13.29 & 0.32 \\
17 & $\mathrm{~T}-2$ & 17.18 & 0.43 \\
33 & $\mathrm{~T}-3$ & 12.85 & 0.30 \\
\hline
\end{tabular}

Tabla 5: Contenido proteico de los granos a partir de los resultados obtenidos mediante el análisis por activación neutrónica, siendo T-3 el control.

Las discrepancias porcentuales mayores en el caso de los resultados obtenidos mediante el método absoluto podrían deberse a que en el presente trabajo no se han tenido en cuenta las contribuciones, a la activación neutrónica de las muestras, de las componentes espectrales a energías menores de los $14 \mathrm{MeV}$.

\section{Conclusiones}

Se realizó la determinación cuantitativa del porcentaje en peso del nitrógeno y del potasio mediante la aplicación combinada del análisis por fluorescencia de rayos $\mathrm{X}$ y el análisis por activación con neutrones de 14 $\mathrm{MeV}$ obteniéndose resultados que están en concordancia con los que se obtienen mediante métodos químicos altamente consumidores de tiempo y en general destructivos.

Se demuestra la factibilidad de uso de las partes no comestibles de la planta para la elaboración de abonos verdes o mixtos utilizando para ello los índices de remanencia de potasio y nitrógeno en dichas partes, una vez concluida la cosecha.

Se demuestra cuantitativamente, a partir del valor nutricional obtenido para los granos analizados, que éste depende del esquema de fertilización adoptado, por consiguiente, dicho resultado puede usarse como referencia para estudios que establezcan la dosificación y tiempo de aplicación de fertilizantes químicos para el cultivo de la quinua.

\section{Agradecimientos}

Los autores desean dejar constancia explícita de su agradecimiento a la Comisión Boliviana de Energía Nuclear, quien suministró las muestras, a la Academia de Ciencias de Hungría por facilitar el uso del equipamiento involucrado, a la Comisión Internacional de Energía Atómica por el auspicio global del Proyecto de Investigación.

\section{Referencias}

[1] F. Brenes, F. Crespo y K. Madrigal; Instituto Centroamericano de Administración de Empresas, INCAE (2002).

[2] L. Vega-Galvez, M. Miranda, J. Vergara, E. Uribe y E. Martınez; J. Sci. Food Agric. 90, 2541 (2010).

[3] J. Sven-Erik; Food Review International 19, 167 (2003).

[4] P. Pasko, H. Barton, P. Zagrodzki, S. Gorinstein, M. Folta y Z. Zachwieja; Food Chem. 115, 994 (2009).

[5] Bioversity International. Nota Conceptual. Quinua.
Celebrando el Año Internacional de la Quinua: Un Futuro Sembrado hace Miles de Años. (2012).

[6] Organización de las Naciones Unidas para la Agricultura y la Alimentación (FAO), Informe sobre utilización de cultivos andinos, Quito (1990).

[7] Quinua y sus derivados; Centro de Promoción Bolivia, CEPROBOL, Dirección de Investigación e Información (2007).

[8] L. Lamothe, S. Srichuwong, B.Reuhs, R. Hamaker; Food Chem. 167, 490 (2015).

[9] W. Rojas, M. Pinto; Informe Técnico Anual 2002/2003 (Año 2) 59-94. IPGRI-IFAD, Fundación PROINPA, Bolivia (2003). 
[10] S. Thanapornpoonpong, S. Vearaslip, E. Pawelzik y S. Gorinstein; Food Chem. 56,11464 (2008).

[11] A. Fravret; Proceeding of a Panel on New Approaches to Breeding for Improved Plant Protein, IAEA (1980).

[12] R. Soto y S. J. Szegedi; Radioanal. Nucl. Chem. Letts. 96, 69 (1985).

[13] R. Soto, S. Szegedi y J. Llópiz; CIEN-R. 7-96. 117. (1996).

[14] J. Ruales y K. Nair; Food Chem. 48, 131 (1993).

[15] J. Ruales ; Inter. J. Food Sci. Nutrition 53, 142 (2002).

[16] H. Morillo; Trabajo de grado, Facultad de Ciencias Agrícolas, Universidad de Nariño, Pasto (2002).

[17] J. Castro; Transformación de los desechos orgánicos en abono orgánico, DELAP, Bolivia (2005).
[18] E. Maturana; Instituto Nacional de Investigación y Extensión Agraria (2004). http://www. consumaseguridad.com/web/es/ investigacion/2004/11/29/15489.php.

[19] D. Hernández, S. Viteri; Agronomía Colombiana 24(1) (2006).

[20] N. Ramos; Tesis de grado, UMSA (2000).

[21] C. Alvarado-Ortiz; artículo publicado en: http://biblioteca.usmp.edu.pe.

[22] B. Guerrero, E. Hernán; Instituto Nacional de Investigaciones Agropecuarias. http://w.w.w.oniap-ecuador.gov.ec/20ISIS.mht

[23] L. Muñoz, A. Lucero; Agronomía Colombiana 26(2), 340 (2008).

[24] F. Crespo,S. Bellot; Perspectivas, Bolivia (2003).

[25] J. Puckett; Quinoa, Post Carbon Institute (2007). http://archive.energyfarms.net/node/1054. 